\title{
Driven optical lattices as strong-field simulators
}

\author{
Stephan Arlinghaus and Martin Holthaus \\ Institut für Physik, Carl von Ossietzky Universität, D-26111 Oldenburg, Germany
}

(Dated: June 21, 2010)

\begin{abstract}
We argue that ultracold atoms in strongly shaken optical lattices can be subjected to conditions similar to those experienced by electrons in laser-irradiated crystalline solids, but without introducing secondary polarization effects. As a consequence one can induce nonperturbative multiphotonlike resonances due to the mutual penetration of ac-Stark-shifted Bloch bands. These phenomena can be detected with a combination of currently available laboratory techniques.

PACS numbers: $67.85 .-\mathrm{d}, 32.80 . \mathrm{Xx}, 42.50 . \mathrm{Hz}$
\end{abstract}

\section{INTRODUCTION}

The investigation of ultracold atoms in optical lattices constitutes a major area of topical research [1-4]. One of the long-term visions driving this trend stems from the prospect of using these well-controllable and flexible systems for "emulating" important quantum many-body problems which still are not fully understood, such as high- $T_{c}$ superconductivity [5, 6], and of obtaining information on these by observing their cold-atom-emulated versions in the laboratory, rather than attempting necessarily imperfect computer simulations. So far, interest has been focused mainly on systems governed by a time-independent Hamiltonian operator, a hallmark example being provided by the Bose-Hubbard model [7]. However, it is feasible to subject the lattice atoms to time-dependent external forces, and thus to study explicitly time-dependent phenomena [8, 9]. Already in 1998 Madison et al. have obtained evidence for Bloch band narrowing with cold sodium atoms in time-periodically forced optical lattices [10]; more recently, dynamic localization 11, 12], photon-assisted tunneling [13], and coherent control of the superfluid-to-Mott insulator transition [14] have been demonstrated with Bose-Einstein condensates in such strongly shaken periodic potentials. Moreover, it has been suggested to employ oscillating optical lattices for realizing frustrated quantum antiferromagnetism [15]. In this article we argue that ultracold atoms in forced optical lattices also lend themselves to the study of multiphoton-like transitions under strongfield conditions which are barely accessible with electrons in solids irradiated by high-power lasers; in particular, they provide an exceptionally clean testing ground for the investigation of nonperturbative multiphoton-like resonances. We first sketch in Sec. II the required setup, and specify the orders of magnitude of the relevant parameters which characterize the optical-lattice analogs of strong laser fields. We then present numerical model calculations in Sec. III, demonstrating how both perturbative and nonperturbative resonances manifest themselves. The explanation of these phenomena makes use of both the spatial periodicity of the optical lattice and the temporal periodicity of the driving force: Effectively, one encounters a spatiotemporal crystal, the band structure of which is controlled by the parameters of the driving force. This viewpoint is emphasized in the concluding Sec. IV

\section{SIMULATING STRONG LASER FIELDS}

A one-dimensional (1D) optical lattice is created, for example, by shining laser radiation with wavelength $\lambda=$ $2 \pi / k_{\mathrm{L}}$ against a mirror and retroreflecting the beam into itself. An atom of mass $M$ moving in this standing light wave then experiences a periodic potential with a depth $V_{0}$ which is proportional to the laser intensity [2]. Mounting the mirror on a piezoelectric actuator now allows one to let it oscillate sinusoidally with a precisely controlled angular frequency $\omega$ and amplitude $L$, thus shaking the lattice back and forth [14]. In the laboratory frame, the Hamiltonian describing the particle's centerof-mass motion along the lattice direction then reads

$$
H_{\text {lab }}=\frac{p^{2}}{2 M}+\frac{V_{0}}{2} \cos \left\{2 k_{\mathrm{L}}[x-L \cos (\omega t)]\right\} .
$$

The relevant characteristic energy scale is given by the single-photon recoil energy,

$$
E_{\mathrm{r}}=\frac{\hbar^{2} k_{\mathrm{L}}^{2}}{2 M}
$$

typical scaled lattice depths $V_{0} / E_{\mathrm{r}}$ range between about 5 and 10 . For example, with ${ }^{87} \mathrm{Rb}$ atoms in a lattice erected by light with wavelength $\lambda=842 \mathrm{~nm}$ one has $E_{\mathrm{r}}=1.34 \times 10^{-11} \mathrm{eV}$, as corresponding to the recoil frequency $\nu_{\mathrm{r}}=E_{\mathrm{r}} /(2 \pi \hbar)=3.23 \mathrm{kHz}$.

Performing a unitary transformation to a frame comoving with the lattice, the Hamiltonian acquires the suggestive form [10, 16]

$$
H=\frac{p^{2}}{2 M}+\frac{V_{0}}{2} \cos \left(2 k_{\mathrm{L}} x\right)-F x \cos (\omega t),
$$

with $F=M L \omega^{2}$ denoting the amplitude of the inertial force appearing in this oscillating frame. A meaningful measure for the strength of this force is the dimensionless parameter 12]

$$
K_{0}=\frac{F d}{\hbar \omega}
$$




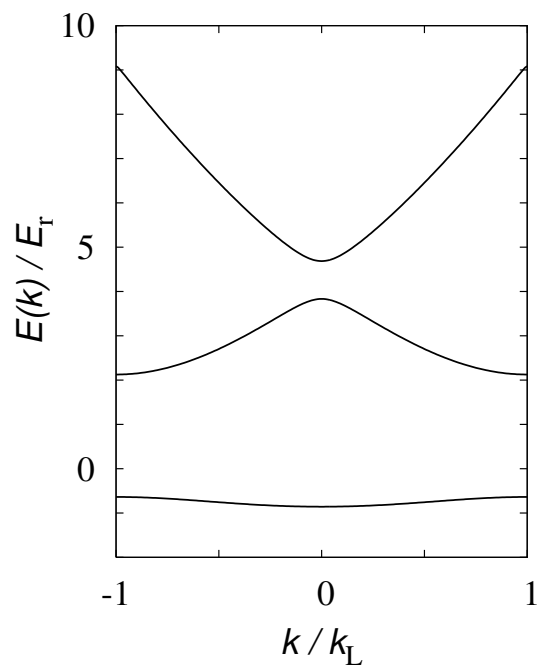

FIG. 1: Lowest three Bloch bands of a 1D optical lattice with depth $V_{0}=5.7 E_{\mathrm{r}}$. The lowest band gap is $2.763 E_{\mathrm{r}}$ at $k=k_{\mathrm{L}}$.

where $d=\lambda / 2$ specifies the lattice constant. In terms of quantities directly accessible in the laboratory, one has

$$
K_{0}=\frac{\pi^{2}}{2} \frac{\nu}{\nu_{\mathrm{r}}} \frac{L}{d}
$$

with the driving frequency $\nu=\omega /(2 \pi)$, showing that one may easily realize values $K_{0}>1$ when both ratios $\nu / \nu_{\mathrm{r}}$ and $L / d$ are on the order of unity [11 14]. To appreciate what this means, consider an atomic analog: A common $\mathrm{KrF}$ exciplex laser provides photons with energy $\hbar \omega=5.0 \mathrm{eV}$. Inserting this into the expression (4), taking the Bohr radius for the length $d$, and solving for the electric field strength $\mathcal{E}=F / e$ acting on an electronic charge, one finds that $K_{0}=1$ is reached only for $\mathcal{E}=9.45 \times 10^{10} \mathrm{~V} / \mathrm{m}$, which is roughly one-fifth of the field formally experienced by a ground-state electron in the hydrogen atom. In this sense, time-periodically forced optical lattices can serve even as superstrong-field simulators: Shaking a lattice with large amplitudes $L$ according to the Hamiltonian (11) simulates perfectly homogeneous fields acting on particles in periodic potentials in the regime $K_{0}>1$ of the parameter (4) which is hard to reach with laser-driven electrons in traditional solids, without introducing, for example, detrimental polarization effects. Thus, ultracold atoms in driven optical lattices offer the unique possibility to study superstrongfield-induced multiphoton-like processes in periodic potentials in their purest form.

\section{PERTURBATIVE AND NONPERTURBATIVE MULTIPHOTON TRANSITIONS}

For illustrating the dynamics that become explorable in this way, we consider a 1D lattice with depth
$V_{0}=5.7 E_{\mathrm{r}} . \quad$ Its single-particle eigenstates are Bloch waves [17],

$$
\varphi_{n, k}(x)=\exp (\mathrm{i} k x) u_{n, k}(x),
$$

with lattice-periodic functions $u_{n, k}(x)=u_{n, k}(x+d)$ labeled by a band index $n$ and a wave number $k$; Fig. 1 depicts the energy dispersion relations $E_{n}(k)$ for the lowest bands $n=1,2,3$. In the center of the Brillouin zone, that is, at $k / k_{\mathrm{L}}=0$, one has $E_{2}(0)-E_{1}(0)=4.690 E_{\mathrm{r}}$, and $E_{3}(0)-E_{1}(0)=5.544 E_{\mathrm{r}}$. We now take an initial state exclusively populating the lowest band, as described by

$$
\psi\left(x, t_{0}\right)=\int_{-k_{\mathrm{L}}}^{+k_{\mathrm{L}}} \mathrm{d} k g_{1}\left(k, t_{0}\right) \varphi_{1, k}\left(x, t_{0}\right)
$$

with a Gaussian $k$-space distribution

$$
g_{1}\left(k, t_{0}\right)=\left(2 k_{\mathrm{L}} \sqrt{\pi} \Delta k\right)^{-1 / 2} \exp \left(-\frac{k^{2}}{2(\Delta k)^{2}}\right)
$$

centered around $k / k_{\mathrm{L}}=0$, and set $\Delta k=0.1 k_{\mathrm{L}}$ for its width, as appropriate for an initial ensemble of noninteracting ultracold atoms. This state then is subjected to pulsed forcing with an amplitude $F(t)$ which rises from zero to a maximum value, stays constant for a while, and decreases back to zero. For the sake of definiteness, we consider conditions as already realized experimentally in Ref. 14]: We take ${ }^{87} \mathrm{Rb}$ as atomic species in a lattice with $\lambda=842 \mathrm{~nm}$, and design the envelope of the pulse such that $F(t)$ rises linearly within $10 \mathrm{~ms}$, stays constant for a holding time of $2 \mathrm{~ms}$, and then is linearly switched off in another $10 \mathrm{~ms}$. For a driving frequency of $5 \mathrm{kHz}$, say, the ramp time of $10 \mathrm{~ms}$ corresponds to 50 cycles, so that the relatively slowly changing envelope $F(t)$ may enable adiabatic following under nonresonant conditions.

Moreover, we rely on the fact that the fraction of atoms surviving in the lowest band can be accurately determined, as demonstrated by the Landau-Zener measurements reported in Ref. [18]. We therefore compute the escape probability from the lowest band after each pulse, for specified values of $K_{0}^{\max }$ reached during the plateau phase. Figure 2 shows results thus obtained for $K_{0}^{\max }=0.7$ and $K_{0}^{\max }=1.3$, as functions of the driving frequency $\omega /(2 \pi)$. The pronounced peak pattern depends markedly on the maximum driving amplitude; for instance, a further peak has appeared for $K_{0}^{\max }=1.3$ at $\omega /(2 \pi) \approx 4 \mathrm{kHz}$ which was not visible for $K_{0}^{\max }=0.7$. A more complete picture is provided by Fig. 3, which shows a two-dimensional plot of the escape probability considered as function of both $\omega /(2 \pi)$ and $K_{0}^{\max }$, for the same pulse shape as taken in Fig. 2 .

The positions of most of the peaks in Figs. 2 and 3 (i.e., most of the system's resonant frequencies) are easily explained: Because the initial state is narrowly centered around $k / k_{\mathrm{L}}=0$, its response is mainly determined by the energies $E_{n}(0)$ in the Brillouin-zone center. Hence, one expects ordinary $m$-photon-like resonances between 


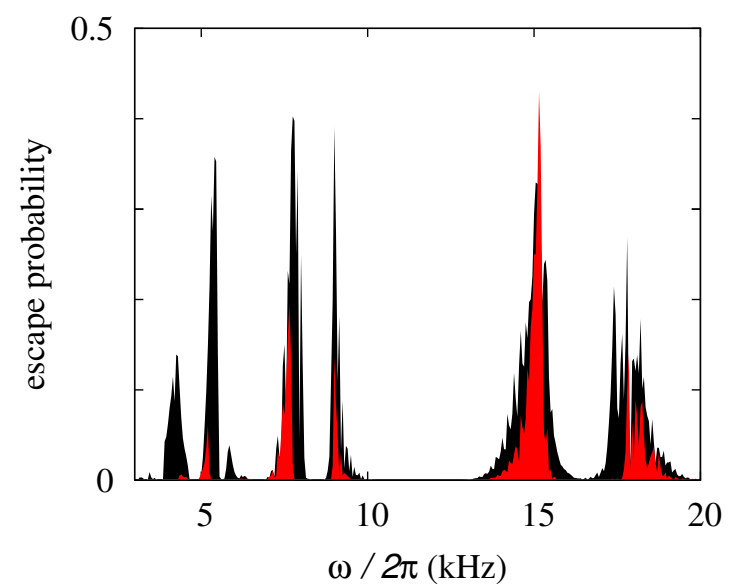

FIG. 2: (Color online) Escape probabilities from the lowest Bloch band after pulses with linear switch-on and switchoff ramps of 10-ms duration each, and a holding time of $2 \mathrm{~ms}$, during which a specified value $K_{0}^{\max }$ of the scaled amplitude (4) is reached. Driving frequencies $\omega /(2 \pi)$ correspond to ${ }^{87} \mathrm{Rb}$ in an optical lattice with $\lambda=842 \mathrm{~nm}$. Light, $K_{0}^{\max }=0.7$; black, $K_{0}^{\max }=1.3$. Of particular interest is the unexpected, strong, and narrow resonance at $\omega /(2 \pi)=5.3 \mathrm{kHz}$.

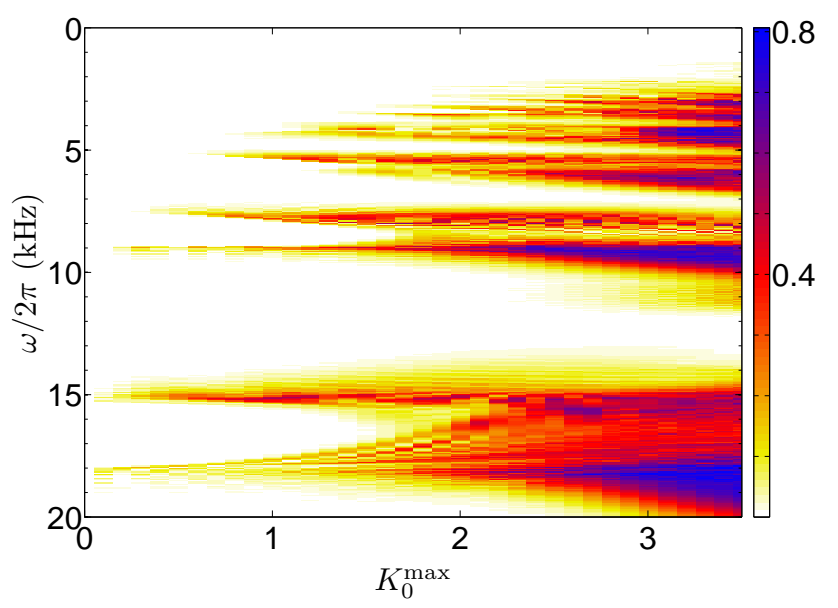

FIG. 3: (Color online) Escape probability versus both driving frequency $\omega /(2 \pi)$ and maximum scaled amplitude $K_{0}^{\max }$, for the same pulse shape as employed in Fig. 2.

the initial band $n=1$ and higher bands $n=2,3, \ldots$ when the driving frequency complies with the condition

$$
\Delta E_{n, 1} \equiv E_{n}(0)-E_{1}(0)=m \hbar \omega
$$

for integer $m$. Indeed, listing these expected $m$-photon transition frequencies in Table $\$ and comparing them to the frequencies of the peaks observed in Fig. 3. one generally finds quite good agreement.

In some instances, however, the numerical solution of the Schrödinger equation produces a peak which does not fit into this naive pattern. Most notably, the sharp spike visible in Fig. 2] at $\omega /(2 \pi)=5.3 \mathrm{kHz}$ does not

\begin{tabular}{ccccc}
\hline \hline$m$ & $n$ & $\Delta E_{n, 1} /\left(m E_{\mathrm{r}}\right)$ & $\nu_{\text {res }}(\mathrm{kHz})$ & $\nu_{\text {peak }}(\mathrm{kHz})$ \\
\hline 1 & 3 & 5.544 & 17.932 & 18.00 \\
1 & 2 & 4.690 & 15.170 & 15.15 \\
2 & 3 & 2.772 & 8.966 & 9.00 \\
2 & 2 & 2.345 & 7.585 & 7.60 \\
3 & 3 & 1.848 & 5.977 & 5.85 \\
& & -- & -- & 5.30 \\
3 & 2 & 1.563 & 5.057 & $\mathrm{NV}$ \\
\hline \hline
\end{tabular}

TABLE I: Expected and computed resonance frequencies: $\nu_{\text {res }}$ is an $m$-photon transition frequency according to Eq. (9), $\nu_{\text {peak }}$ is the position of the corresponding peak where it becomes apparent in Fig. 3. The entry NV indicates that no peak is visible for the pulse profile employed here.


FIG. 4: Quasienergies $\varepsilon_{n}(k)$ for the $1 \mathrm{D}$ optical lattice driven with frequency $\omega /(2 \pi)=5.30 \mathrm{kHz}$, and scaled amplitudes $K_{0}=0.7$ (a), $1.0(\mathrm{~b})$, and 1.3 (c). The areas shaded in gray, extending from $k=-0.1 k_{\mathrm{L}}$ to $k=+0.1 k_{\mathrm{L}}$, mark the range of wave numbers explored by the initial wave packet. The insets show how the quasienergy band $n=1$ (above) is pinched through with increasing $K_{0}$ by the band $n=2$, displaced downward by $3 \hbar \omega$. This causes the nonperturbative resonance observed in Fig. 2 .

match Eq. (9) for any reasonable combination of $n$ and $m$. Such "nonperturbative" events are our main concern; we predict that they can be detected experimentally in already existing setups. These particular resonances admit a systematic explanation which forces us to go way beyond the perturbative reasoning underlying Eq. (9).

Because the Hamiltonian (11) is periodic both in space (with lattice period $d=\pi / k_{\mathrm{L}}=\lambda / 2$ ) and in time (with 
driving period $T=2 \pi / \omega)$, it gives rise to spatiotemporal Bloch waves [16],

$$
\psi_{n, k}(x, t)=u_{n, k}(x, t) \exp \left\{\mathrm{i}\left[k x-\varepsilon_{n}(k) t / \hbar\right]\right\},
$$

with functions $u_{n, k}(x, t)=u_{n, k}(x+d, t)=u_{n, k}(x, t+T)$ reflecting translational invariance in space and time on equal footing, and quasienergies $\varepsilon_{n}(k)$, in generalization of the usual Bloch waves (6). While quasimomenta $\hbar k$ are determined up to an integer multiple of $2 \pi \hbar / d=2 \hbar k_{\mathrm{L}}$, quasienergies are likewise determined up to an integer multiple of the photon energy $2 \pi \hbar / T=\hbar \omega$. Figure 4 shows one "quasienergy Brillouin zone" (of height $\hbar \omega$ ) with states originating from the lowest three Bloch bands for $\omega /(2 \pi)=5.30 \mathrm{kHz}$, the frequency of the extraordinary peak in Fig. 2 and $K_{0}=0.7,1.0$, and 1.3. There are various avoided crossings indicating multiphoton-like couplings between the bands; however, with $\Delta k=0.1 k_{\mathrm{L}}$ the wave packet evolving from the initial distribution (8) mainly explores the interval of quasimomenta indicated by the shaded areas. The quasienergy band originating from the lowest unperturbed energy band $n=1$ is shown enlarged in the insets; with increasing $K_{0}$ this band is pierced through from below by the quasienergy band $n=2$, displaced down in energy by $3 \hbar \omega$ against that representative which is continuously connected to the bare $n=2$ Bloch band. This penetration results in "active" avoided crossings signaling a strong-field-induced threephoton resonance; this is responsible for the anomalous peak at $\omega /(2 \pi)=5.30 \mathrm{kHz}$.

The dynamics underlying that peak should thus be discussed in terms of the morphology of the surfaces which emerge when the quasienergies are considered as functions of both the wave number $k$ and the instantaneous amplitude $F$ (or $K_{0}$ ): When the driving amplitude $F(t)$ increases during the upward ramp of a pulse, the initial distribution is shifted almost adiabatically on its quasienergy surface, parallel to the $K_{0}$ axis. As long as the maximum value of $K_{0}$ lies below the critical regime where this surface is first being pierced by another one, the initial distribution is restored with only minor distortion when the amplitude returns to zero, resulting in negligible escape probability. However, when the moving distribution hits an avoided-crossing regime, part of the wave function undergoes a Landau-Zener-type transition to the anticrossing band. Both parts of the wave function then evolve separately on their respective surfaces, until they meet for a second time during the downward ramp, when they interfere and thereby establish the final occupation probabilities of the bands involved. This mechanism of splitting and interference implies that there should be Stückelberg-like oscillations when the final occupation probabilities are monitored while the length of the pulses' plateau segment is varied, because varying the plateau duration means varying the relative phase picked up by the two interfering components. Indeed, these oscillations are clearly visible in Fig. 5 .

We remark that the standard perturbative $m$-photon resonances can be grasped in a similar manner: For fre-

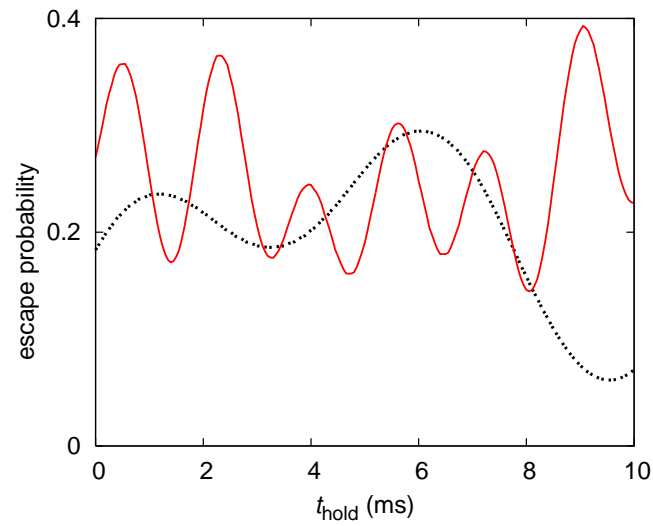

FIG. 5: (Color online) Stückelberg oscillations of the escape probability in response to prolongation of the plateau duration $t_{\text {hold }}$, for $\omega /(2 \pi)=5.30 \mathrm{kHz}$, and $K_{0}^{\max }=1.0$ (dashed) and 1.3 (solid line).

quencies such that Eq. (9) holds, two quasienergy surfaces are degenerate already at $F=0$, so that adiabaticity is disabled and the wave function splits right at the beginning of a pulse [19]. Seen against this background, a perturbative resonance corresponds to the removal of a quasienergy degeneracy already present at $F=0$, while a nonperturbative one emerges when acStark-shifted Bloch bands penetrate each other at a certain finite driving strength.

\section{CONCLUSIONS}

When viewing a time-periodically forced optical lattice as a spatiotemporal crystal, the natural basis states are the spatiotemporal Bloch waves (10); the energy bands $E_{n}(k)$ of the undriven lattice turn into quasienergy bands $\varepsilon_{n}(k)$. The latter depend not only on the lattice parameters, but also on the parameters of the driving force. While they differ barely from the unperturbed energy bands as long as the driving amplitude is weak, corresponding to values $K_{0} \ll 1$ of the dimensionless parameter (4), they become strongly distorted, and even penetrate each other, in the nonperturbative regime.

When subjected to pulsed forcing with an amplitude which changes slowly compared to the period $T=2 \pi / \omega$ of the drive, a wave packet can adjust itself adiabatically to a mere distortion of its quasienergy band. However, when the wave packet explores a part of a quasienergy band which is pierced by another one, as exemplified in Fig. 4. Landau-Zener transitions occur; this mechanism leads to strong nonperturbative resonances at frequencies not given by the simple condition (9). In principle, such resonances should also occur in solids irradiated by strong laser pulses; however, there they would be masked by a host of competing effects. The experimentally proven good controllability of ultracold atoms in forced optical lattices makes such systems a far better testing ground 
for these dynamics.

Our study has been restricted to the single-particle level; it is reasonable to expect that the phenomena exemplarily discussed in the present work can immediately be detected with sufficiently dilute or close-to-ideal BoseEinstein condensates in driven optical lattices [12]. Even more, it appears equally feasible to perform the experiments suggested here under conditions of sizable interparticle interactions, or even of strong correlations. The question how the single-particle scenario outlined above is modified then opens up far-reaching further lines of investigation, concerning both experiment and theory.

\section{Acknowledgments}

We thank Oliver Morsch for continuing in-depth discussions of the Pisa experiments [11 14, 18]. This work was supported by the Deutsche Forschungsgemeinschaft under Grant No. Ho 1771/6.
[1] D. Jaksch and P. Zoller, Ann. Phys. (N.Y.) 315, 52 (2005).

[2] O. Morsch and M. Oberthaler, Rev. Mod. Phys. 78, 179 (2006).

[3] M. Lewenstein, A. Sanpera, V. Ahufinger, B. Damski, A. Sen, and U. Sen, Adv. Phys. 56, 243 (2007).

[4] I. Bloch, J. Dalibard, and W. Zwerger, Rev. Mod. Phys. 80, 885 (2008).

[5] W. Hofstetter, J. I. Cirac, P. Zoller, E. Demler, and M. D. Lukin, Phys. Rev. Lett. 89, 220407 (2002).

[6] I. Bloch, Science 319, 1202 (2008).

[7] M. Greiner, O. Mandel, T. Esslinger, T. W. Hänsch, and I. Bloch, Nature 415, 39 (2002).

[8] S. D. Huber, E. Altman, H. P. Büchler, and G. Blatter, Phys. Rev. B 75, 085106 (2007).

[9] C. Gaul, R. P. A. Lima, E. Díaz, C. A. Müller, and F. Domínguez-Adame, Phys. Rev. Lett. 102, 255303 (2009).

[10] K. W. Madison, M. C. Fischer, R. B. Diener, Q. Niu, and M. G. Raizen, Phys. Rev. Lett. 81, 5093 (1998).

[11] H. Lignier, C. Sias, D. Ciampini, Y. Singh, A. Zen- esini, O. Morsch, and E. Arimondo, Phys. Rev. Lett. 99, 220403 (2007).

[12] A. Eckardt, M. Holthaus, H. Lignier, A. Zenesini, D. Ciampini, O. Morsch, and E. Arimondo, Phys. Rev. A 79, 013611 (2009).

[13] C. Sias, H. Lignier, Y. P. Singh, A. Zenesini, D. Ciampini, O. Morsch, and E. Arimondo, Phys. Rev. Lett. 100, 040404 (2008).

[14] A. Zenesini, H. Lignier, D. Ciampini, O. Morsch, and E. Arimondo, Phys. Rev. Lett. 102, 100403 (2009).

[15] A. Eckardt, P. Hauke, P. Soltan-Panahi, C. Becker, K. Sengstock, and M. Lewenstein, EPL 89. 10010 (2010).

[16] K. Drese and M. Holthaus, Chem. Phys. 217, 201 (1997).

[17] N. W. Ashcroft and N. D. Mermin, Solid State Physics (Harcourt College Publishers, Fort Worth etc., 1976).

[18] A. Zenesini, H. Lignier, G. Tayebirad, J. Radogostowicz, D. Ciampini, R. Mannella, S. Wimberger, O. Morsch, and E. Arimondo, Phys. Rev. Lett. 103, 090403 (2009).

[19] M. Holthaus and B. Just, Phys. Rev. A 49, 1950 (1994). 